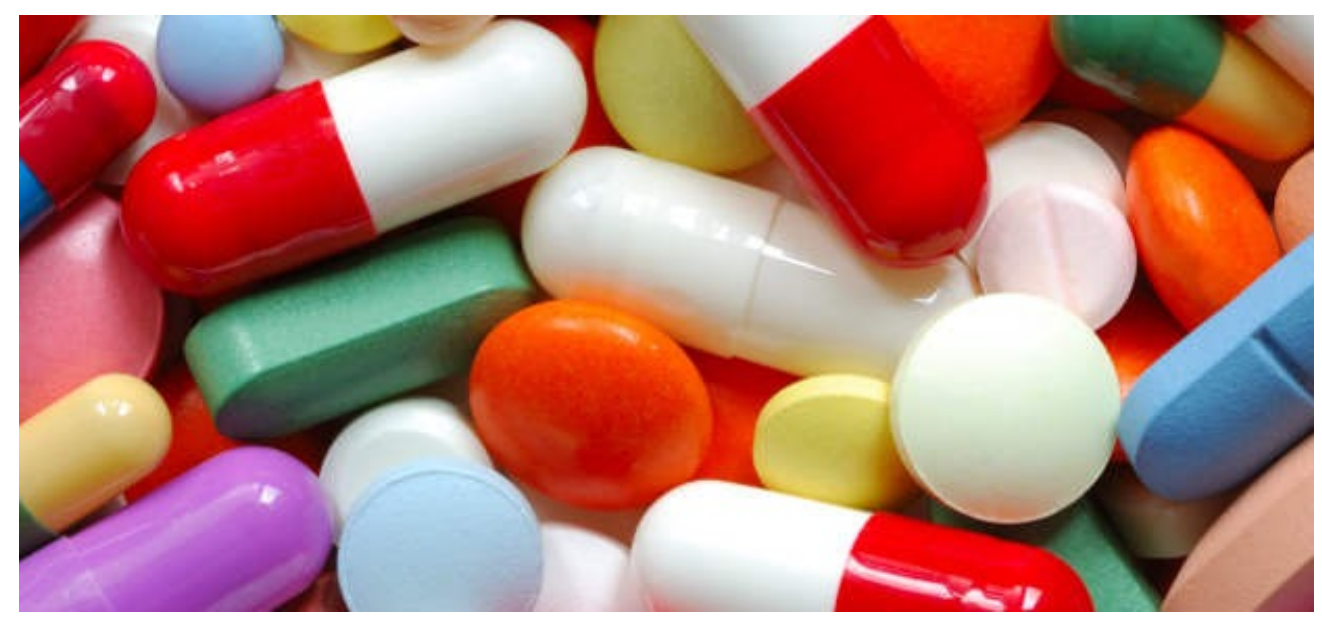

\title{
Anti-inflammation Effects of Cupping Therapy in Cancer
}

\author{
Nooshin Abbasi $^{1}$, rezvan najafi ${ }^{2}$ \\ 1 University of Padua \\ 2 Hamadan University of Medical Sciences
}

Funding: The author(s) received no specific funding for this work.

Potential competing interests: The author(s) declared that no potential competing interests exist.

\section{Abstract}

Studies have demonstrated that chronic, persistent and unresolved inflammation predisposes individuals to almost $20 \%$ of cancers. Both tumor and immune cells secrete inflammatory mediators that promote tumor initiation and progression. Inhibiting or modifying tumor cytokine network and signaling may result in systematic or tumor-specific therapeutic effects. Cupping therapy, as an ancient therapeutic method, is mainly practiced to improve quality of life and to treat aches and pains in head, neck, shoulders and back areas. However, anti-inflammatory and immunomodulatory effects of cupping therapy have been proved in different pathological conditions rather than only for aches and pains, but no study has yet assessed the effects of cupping therapy in cancer. We hypothesize that cupping therapy may improve the efficacy of conventional therapeutics and immunotherapies by preventing or delaying cancer onset. This study focuses on the anti-inflammatory and immunomodulatory effects of cupping therapy in different pathological conditions and suggests that scientists and clinicians assess the effects of cupping therapy as an adjuvant treatment along with other therapeutic strategies to prevent and cure cancer. 


\section{Introduction}

Inflammation plays a pivotal role in tumor initiation, promotion, malignant conversion, invasion, and metastasis. An inflammatory microenvironment is an essential component to almost all tumors. Different forms of chronic inflammation, classifying by cause, mechanism, outcome, and intensity, are known as risk factors to cause cancer ${ }^{[1]}$. Epidemiological and molecular studies have demonstrated that chronic, persistent and unresolved inflammation predisposes individuals to almost $20 \%$ of cancers including: microbial infections in gastric cancer and gastric mucosal lymphoma, inflammatory conditions of unknown origin in prostate cancer, exposure to irritants and autoimmune disease in colon cancer, which is a type of chronic inflammation caused by immune deregulation and autoimmunity ${ }^{[2][3][4]}$.

Tumor and immune cells secrete inflammatory mediators such as cytokines and chemokines within the tumor microenvironment; these mediators stimulate the epithelial to mesenchymal transition (EMT) and metastasis ${ }^{[5][6]}$. Inhibiting or modifying tumor cytokine network and signaling may result in a systematic or tumor-specific therapeutic effects ${ }^{[7]}$.

\section{The Hypothesis}

The existence of inflammatory cells and mediators of innate immune system such as chemokines, cytokines and prostaglandins are detected in the microenvironment of most tumors that have not even demonstrated a certain relationship or epidemiological basis to inflammation ${ }^{[3]}$. In addition, genetic alterations in dominant oncogenes cause the development of a human tumor and create an inflammatory microenvironment ${ }^{[8]}$. Genetic alteration in RET involves interleukin $1 \beta$ (IL-1 $\left.\beta\right)$, colony-stimulating factors (CSFs), cyclooxygenase 2 (COX-2), CC-chemokine ligand 2 (CCL2), CCL20, IL-8, the chemokine receptor CXC-chemokine receptor 4 (CXCR4), extracellular-matrix-degrading enzymes and the adhesion molecule lymphocyte selectin (L-selectin) ${ }^{[8][9]}$; and genetic alteration in MYC involves the production of several chemokines that recalls mast cells together with the production of inflammatory cytokine IL-1 $\beta$ cause angiogenesis and tumor growth ${ }^{[10]}$.

Pre-existing inflammations induce carcinogenesis, but also cancer leads to inflammation thus antiinflammation therapy may be seen as a promising approach to prevent and cure cancers ${ }^{[7]}$. Cupping therapy is an ancient therapeutic method practiced mainly in the Middle East and China. Cupping improves the quality of life by applying quick, vigorous, rhythmical strokes to stimulate muscles and to treat aches and pains in head, neck, shoulders and back areas ${ }^{[11]}$.

However anti-inflammatory and immunomodulatory effects of cupping therapy as a part of complementary and alternative medicine (CAM) have been proved in different pathological conditions rather than only aches and pains, but no study has yet assessed the effects of cupping therapy in cancer.

We hypothesize that cupping therapy may improve the efficacy of conventional therapeutics and immunotherapies by preventing or delaying cancer onset. This study focuses on the anti-inflammatory and immunomodulatory effects of cupping therapy in different pathological conditions and suggests that scientists and clinicians assess the effects of cupping therapy as an adjuvant treatment along with other therapeutic strategies to prevent and cure cancer. 


\section{Evaluation of the hypothesis}

Cupping therapy, as an old medical therapy, has been mentioned by Herodotus (a Greek historian, 400 BC) and Hippocrates in their prescriptions and in the Egyptian Papyrus Ebers (1550 BC) to cure different pathological conditions such as headaches, lack of appetite, maldigestion, fainting, abscess evacuation, narcolepsy musculoskeletal diseases, gynecological complaints, pharyngitis, ear ailments, and lung diseases $^{[12]}$. Aretaeus applied two main cupping therapy methods, wet and dry cupping, to treat prolapse of the uterus, cholera ileus and epilepsy in the early 2 nd century $(\mathrm{CE})^{[11]}$.

Under different pathological conditions, the cups are placed on different parts of the body skin and generally in areas of abundant muscle including back, chest, abdomen, and buttock ${ }^{[13]}$. A negative pressure is applied for about 5 to 10 minutes using a flame to remove oxygen or through connecting a suction device to the cup. In application sites histological changes, vasodilatation and edema are observed $^{[11]}$. In wet cupping, the skin under the cup area is scarified, and blood flows into the cup following the second suction.

The effects of cupping therapy on immune system and inflammation

Cupping therapy, as a neuroendocrine-immunomodulator, stimulates the body surface and makes changes in microenvironment of stimulated area through external factors, including negative pressure and cuts, and internal factors such as endogenous changes in $\mathrm{pH}$, blood flow, oxygen, secreted cytokines and neurotransmitters, and immune cells function in particular mast cells activation level ${ }^{[14][15]}$. However, the exact mechanism of cupping therapy has not been identified yet but modern medicine proposes several theories and offers possible explanations for its effects on pain management, muscle relaxation, immunological and hormonal adjustments, and waste removal. These theories include "Pain-Gate Theory", "Diffuse Noxious Inhibitory Controls" and "Reflex zone theory" to manage pain and to explain biomechanical changes in the skin, "Nitric Oxide theory" to relax muscles and to explain increased blood circulation, "Activation of immune system theory" to immunomodulate and to adjust hormones, and "Blood Detoxification Theory" to remove toxins from blood and interstitial fluids ${ }^{[16]}$. Another theory that explains the mechanism of cupping therapy is suggested by El Sayed SM Mahmoud HS in (2013). This theory called Taibah, suggests that wet cupping acts very similar to an artificial kidney and performs a percutaneous excretory and physiological principle under the cups area through pressure dependent and size dependent filtration of hydrophilic and hydrophobic materials as lipoproteins ${ }^{[17][18]}$. However, kidney filters hydrophobic materials only through the glomeruli via normal pressure filtration, but wet cupping filters both hydrophilic and hydrophobic material through high-pressure filtration to clear blood and fluids from pathological substances ${ }^{[19]}$.

Pro- and anti-inflammatory lipids Polyunsaturated fatty acids (PUFAs) are essential to initiate or to eliminate inflammation. Lipids play a key 
factor in homeostasis and some physiological conditions ${ }^{[20][21]}$. Several inflammatory human diseases such as diabetes, atherosclerosis, asthma, and arthritis may benefit from anti-inflammatory activity of $\omega-3$ PUFAs ${ }^{[22]}$. The lack of $\omega-3$ PUFAs leads to some chronic inflammatory diseases, including obesity and diabetes $^{[23]}$. Qi Zhanga and colleagues in 2018 constructed a cupping mice model and analyzed lipid metabolism and quantified fatty acids in skin or plasma of nude mice before and after applying wet cupping therapy. They assessed the regulatory effects of wet cupping therapy on the polyunsaturated fatty acids (PUFAs) metabololipidome. The analyses showed that wet cupping therapy could increase antiinflammatory lipids and reduce pro-inflammatory lipids in both skin and plasma. This study identified that wet cupping therapy reduces the secretion of IL- 6 and TNF- $\alpha$ induced by lipopolysaccharide (LPS) in vivo and showed that cupping treatment modulates the metabolic balance between pro- and anti-inflammatory PUFAs ${ }^{[20]}$.

Preclinical studies showed that statins, as cholesterol lowering drugs, prevent angiogenesis and inflammation ${ }^{[24]}$. They prevent the conversion of HMG-CoA, an ER integral membrane protein and the major rate-limiting enzyme of the mevalonate pathway, to mevalonate ${ }^{[25]}$. Mevalonate, the 6 -carbon product formed by HMG-COA, is crucial in membrane integrity, cell signaling, protein synthesis, and cell cycle progression. Statins inhibit this conversion in tumor cells, disrupt tumor initiation, growth and metastasis, and finally lead to cancer cell apoptosis ${ }^{[25]}$. The prolonged cholesterol lowering effect of statins, may have anti- tumorigenic functions through downregulation or inhibition of matrix metalloproteinases (MMPs), which is involved in tumor growth, invasion, and metastasis ${ }^{[26]}$. Similar to statins, cupping therapy showed to be effective method to reduce LDL. Wet cupping therapy has a systematic effect to improve lipid metabolism through reducing total cholesterol, triglyceride and LDL concentrations $^{[27]}$. Analyzing cupping blood revealed a higher concentration of LDL cholesterol and triglycerides in the cupping blood than the blood taken directly from veins ${ }^{[28][29]}$.

\section{Th1/Th2 and Treg/Th17 ratios}

Reza Soleimani and colleagues in an observational study on healthy individuals assessed the immunomodulatory effects of wet cupping therapy and investigated the regulatory effects of this commonly used procedure on the transcription factors of T-lymphocyte subsets and its role to reduce inflammation. They found higher Foxp3 (Treg), GATA-3 (Th2) gene expressions, higher Foxp-3/RORyt (Treg)/ (Th17) gene expression ratio, and lower Tbet/GATA-3 (Th1/Th2) gene expression ratio after cupping treatment. A higher Treg/Th17 ratio indicates a higher immunologic tolerance, which defends body from autoimmune diseases. A lower expression of Tbet means a lower number of inflammatory cytokines produced by Th1 in the body. A lower Th1/Th2 indicates the suppression of inflammation in the body and health improvement. Their results illustrated that wet cupping therapy may treat or reduce warm inflammation symptoms by increasing Th2 and Treg cells and decreasing Th1 and Th17 ${ }^{[30]}$. 
The cyclooxygenase COX-2 enzymes catalyze the conversion of arachidonic acid into prostaglandins (PGs) ${ }^{[31]}$. PGs inhibit cancer cells apoptosis, enhance cancer cell migration and promote neoangiogenesis in stromal tissue ${ }^{[32]}$. A higher level of COX-2 is detected in breast, prostate, pancreas, skin, lung, bladder and head and neck cancers ${ }^{[33][34][35][36][37][38][39]}$. Anti-inflammatory agents including NSAIDs, aspirin and statins used to treat other diseases are seen to be effective in treatment of cancer ${ }^{[7]}$.

Cupping therapy may also be seen as a novel method to excrete PGs from the blood capillaries. The negative pressure created into the cup, from 150 to $420 \mathrm{mmHg}$ and the scratches made with scalpel stimulates innate immune system, inflammatory cell migration and endogenous opioid release ${ }^{[40]}$. The suction increases blood volume under the cup area and increases capillary filtration rate. When the cuts are applied, the negative pressure excretes prostaglandins and inflammatory mediators from the blood and interstitial fluid. Cupping therapy improves blood flow, oxygen supply and tissue perfusion ${ }^{[18]}$. A randomized clinical trial on sixty-six patients assessed the effects of wet cupping therapy to reduce pressure and pain and to improve joint movements in acute scapulohumeral periarthritis. They tested 5hydroxy-tryptamine (5-HT) and prostaglandin $\mathrm{E}_{2}\left(\mathrm{PGE}_{2}\right)$ of blood in the cups and demonstrated the excretion of these inflammatory substances in local blood of the affected shoulder through wet cupping therapy. They showed a decrease in $5-\mathrm{HT}$ and $\mathrm{PGE}_{2}$ in the body and an improvement in pain and shoulder movements after applying $\mathrm{EA}^{[41]}$.

\section{Conclusion}

Similarly to anti-inflammatory drugs, cupping therapy may be seen as an adjuvant therapeutic strategy to modulate host microenvironment by reducing inflammation and modulating immune system, all actions that could be useful in biological treatments, in cancer patients. However, the exact mechanism of action of this ancient therapeutic method is not fully understood yet but we hypothesize that cupping therapy may improve the efficacy of conventional therapeutics and immunotherapies by preventing or delaying cancer onset. We suggest that scientists and clinicians assess the therapeutic effects of cupping therapy as adjuvant treatment along with other therapeutic strategies in treatment of cancer.

\section{References}

1. `Sergei I. Grivennikov, Florian R. Greten, Michael Karin. (2010). Immunity, Inflammation, and Cancer. Cell, vol. 140 (6), 883-899. doi:10.1016/j.cell.2010.01.025.

2. IJ KUNDU, Y SURH. (2008). Inflammation: Gearing the journey to cancer. Mutation Research/Reviews in Mutation Research, vol. 659 (1-2), 15-30. doi:10.1016/j.mrrev.2008.03.002.

3. a, bAlberto Mantovani, Paola Allavena, Antonio Sica, Frances Balkwill. (2008). Cancer-related inflammation. Nature, vol. 454 (7203), 436-444. doi:10.1038/nature07205. 
4. 'Maximilian J. Waldner, Markus F. Neurath. (2009). Colitis-associated cancer: the role of T cells in tumor development. Semin Immunopathol, vol. 31 (2), 249-256. doi:10.1007/s00281-009-0161-8.

5. `Giovanni Germano, Paola Allavena, Alberto Mantovani. (2008). Cytokines as a key component of cancer-related inflammation. Cytokine, vol. 43 (3), 374-379. doi:10.1016/j.cyto.2008.07.014.

6. 'Douglas Hanahan, Lisa M. Coussens. (2012). Accessories to the Crime: Functions of Cells Recruited to the Tumor Microenvironment. Cancer Cell, vol. 21 (3), 309-322. doi:10.1016/j.ccr.2012.02.022.

7. a, b, cShanthini M. Crusz, Frances R. Balkwill. (2015). Inflammation and cancer: advances and new agents. Nat Rev Clin Oncol, vol. 12 (10), 584-596. doi:10.1038/nrclinonc.2015.105.

8. a, bM. G. Borrello, L. Alberti, A. Fischer, D. Degl'Innocenti, et al. (2005). Induction of a proinflammatory program in normal human thyrocytes by the RET/PTC1 oncogene. Proceedings of the National Academy of Sciences, vol. 102 (41), 14825-14830. doi:10.1073/pnas.0503039102.

9. ^V. De Falco, V. Guarino, E. Avilla, M. D. Castellone, et al. (2007). Biological Role and Potential Therapeutic Targeting of the Chemokine Receptor CXCR4 in Undifferentiated Thyroid Cancer. Cancer Research, vol. 67 (24), 11821-11829. doi:10.1158/0008-5472.can-07-0899.

10. ^Laura Soucek, Elizabeth R Lawlor, Darya Soto, Ksenya Shchors, et al. (2007). Mast cells are required for angiogenesis and macroscopic expansion of Myc-induced pancreatic islet tumors. Nat Med, vol. 13 (10), 1211-1218. doi:10.1038/nm1649.

11. a, b, cPiyush Mehta, Vividha Dhapte. (2015). Cupping therapy: A prudent remedy for a plethora of medical ailments. Journal of Traditional and Complementary Medicine, vol. 5 (3), 127-134. doi:10.1016/j.jtcme.2014.11.036.

12. `TurkJL et al.. , , , , et al. (1983). Bleeding and cupping. Ann R Coll Surg Engl.

13. `Simon S. Yoo, Francisco Tausk. (2004). Cupping: East meets West. Int J Dermatol, vol. 43 (9), 664-665. doi:10.1111/j.1365-4632.2004.02224.x.

14. `Yu-Ying Tian et al.. , , , , et al. (2013). [Impacts on skin blood flow under moving cupping along meridians in different directions]. Zhongguo Zhen Jiu.

15. 'Ting Li, Yaoxian Li, Yu Lin, Kai Li. (2016). Significant and sustaining elevation of blood oxygen induced by Chinese cupping therapy as assessed by near-infrared spectroscopy. Biomed. Opt. Express, vol. 8 (1), 223. doi:10.1364/boe.8.000223.

16. A Abdullah M.N. Al-Bedah, Ibrahim S. Elsubai, Naseem Akhtar Qureshi, Tamer Shaban Aboushanab, et al. (2019). The medical perspective of cupping therapy: Effects and mechanisms of action. Journal of Traditional and Complementary Medicine, vol. 9 (2), 90-97. doi:10.1016/j.jtcme.2018.03.003.

17. 'Layla A. Mustafa, Rukzan M. Dawood, Osama M. Al-Sabaawy. (2012). Effect of Wet Cupping on Serum Lipids Profile Levels of Hyperlipidemic Patients and Correlation with some Metal lons. RJS, vol. 23 (5), 128-136. doi:10.33899/rjs.2012.60009.

18. a, boshanak Ghods, Nasrin Sayfouri, Mohammad Hossein Ayati. (2016). Anatomical Features of the Interscapular Area Where Wet Cupping Therapy Is Done and Its Possible Relation to Acupuncture Meridians. Journal of Acupuncture and Meridian Studies, vol. 9 (6), 290-296. 
doi:10.1016/j.jams.2016.06.004.

19. `El Sayed SM Mahmoud HS. (2013). Medical and Scientific Bases of Wet Cupping Therapy (Al-hijamah): in Light of Modern Medicine and Prophetic Medicine. Altern Integ Med, vol. 02 (05). doi:10.4172/23275162.1000122.

20. a, bi Zhang, Xiang Wang, Guifang Yan, Juan Lei, et al. (2018). Anti- Versus Pro-Inflammatory Metabololipidome Upon Cupping Treatment. Cell Physiol Biochem, vol. 45 (4), 1377-1389. doi:10.1159/000487563.

21. `Qi Zhang, Bo Zhu, Yongsheng Li. (2017). Resolution of Cancer-Promoting Inflammation: A New Approach for Anticancer Therapy. Front. Immunol., vol. 8 . doi:10.3389/fimmu.2017.00071.

22. `Yiqing Yan, Wei Jiang, Thibaud Spinetti, Aubry Tardivel, et al. (2013). Omega-3 Fatty Acids Prevent Inflammation and Metabolic Disorder through Inhibition of NLRP3 Inflammasome Activation. Immunity, vol. 38 (6), 1154-1163. doi:10.1016/j.immuni.2013.05.015.

23. `Michael J. Zhang, Matthew Spite. (2012). Resolvins: Anti-Inflammatory and Proresolving Mediators Derived from Omega-3 Polyunsaturated Fatty Acids. Annu. Rev. Nutr., vol. 32 (1), 203-227. doi:10.1146/annurev-nutr-071811-150726.

24. 'Jozef Dulak, Alicja Jozkowicz. (2005). Anti-Angiogenic and Anti-Inflammatory Effects of Statins: Relevance to Anti-Cancer Therapy. CCDT, vol. 5 (8), 579-594. doi:10.2174/156800905774932824.

25. a, buyuhiko Tamanoi, Mahshid Azizian, Mahboobeh Ashrafi, Seyedeh Bathaie. (2017). Mevalonate Pathway and Human Cancers. CMP, vol. 10 (2), 77-85. doi:10.2174/1874467209666160112123205.

26. `Ferdinando Mannello, Gaetana A. Tonti. (2009). Statins and Breast Cancer: May Matrix Metalloproteinase be the Missing Link. Cancer Investigation, vol. 27 (4), 466-470. doi:10.1080/07357900802491444.

27. `Ranaei-siadat S et al.., , , , , et al. (2010). The effect of cupping (hejamat) on blood biochemical and immunological parameters. Iranian Journal of Pharmaceutical Research, vol. 3 .

28. `Majid Niasari, Farid Kosari, Ali Ahmadi. (2007). The Effect of Wet Cupping on Serum Lipid Concentrations of Clinically Healthy Young Men: A Randomized Controlled Trial. The Journal of Alternative and Complementary Medicine, vol. 13 (1), 79-82. doi:10.1089/acm.2006.4226.

29. A Alshowafi FK. , , , , et al. (2010). Effect of Blood Cupping on Some Biochemical Parameter. Med J Cairo Univ.

30. ^Reza Soleimani et al.. , , , , et al. (2019). Comparison of Th1/Th2 and Treg/Th17 ratios between wet and dry cupping therapies in Persian medicine. Avicenna J Phytomed, vol. 10 .

31. ^Rikke Nørregaard, Tae-Hwan Kwon, Jørgen Frøkiær. (2015). Physiology and pathophysiology of cyclooxygenase-2 and prostaglandin E2 in the kidney. Kidney Research and Clinical Practice, vol. 34 (4), 194-200. doi:10.1016/j.krcp.2015.10.004.

32. `Jaime L. Masferrer et al.. , , , , et al. (2000). Antiangiogenic and Antitumor Activities of Cyclooxygenase2 Inhibitors. Cancer Res.

33. `Daniel Hwang, Jane Byrne, David Scollard, Edward Levine. (1998). Expression of Cyclooxygenase-1 and 
Cyclooxygenase-2 in Human Breast Cancer, doi:10.1093/jnci/90.6.455.

34. `Sanjay Gupta, Mayank Srivastava, Nihal Ahmad, David G. Bostwick, et al. (2000). Over-expression of cyclooxygenase-2 in human prostate adenocarcinoma. Prostate, vol. 42 (1), 73-78. doi:10.1002/(sici)1097-0045(20000101)42:1<73::aid-pros9>3.0.c0;2-g.

35. ^Olga N. Tucker et al.. , , , , et al. (1999). Cyclooxygenase-2 Expression Is Up-Regulated in Human Pancreatic Cancer. Cancer Res.

36. ^S. Buckman. (1998). COX-2 expression is induced by UVB exposure in human skin: implications for the development of skin cancer. doi:10.1093/carcin/19.5.723.

37. ^H Wolff et al.. , , , , et al. (1998). Expression of cyclooxygenase-2 in human lung carcinoma. Cancer Res.

38. `Sulma Mohammed, Richard Foster, Nasir Khan, Alane Koki, et al. (1999). EXPRESSION OF CYCLOOXYGENASE (COX) -1 AND COX-2 IN HUMAN INVASIVE TRANSITIONAL CELL CARCINOMA OF THE URINARY BLADDER. The Journal of Urology. doi:10.1097/00005392-199904020-00268.

39. ^G Chan et al.. , , , , et al. (1999). Cyclooxygenase-2 expression is up-regulated in squamous cell carcinoma of the head and neck. Cancer Res.

40. `Salah Mohamed El Sayed, Ashraf Abou-Taleb, Hany Salah Mahmoud, Hussam Baghdadi, et al. (2014). Percutaneous excretion of iron and ferritin (through Al-hijamah) as a novel treatment for iron overload in beta-thalassemia major, hemochromatosis and sideroblastic anemia. Medical Hypotheses, vol. 83 (2), 238-246. doi:10.1016/j.mehy.2014.04.001.

41. `Sun $\mathrm{H}$ et al.. , , , , et al. (2016). Clinical observation of blood-letting to reduce pressure plus electroacupuncture for acute scapulohumeral periarthritis. Zhongguo Zhen Jiu. 